\title{
The effect of attention loading on the inhibition of choice reaction time to visual motion by concurrent rotary motion
}

\author{
MARCIA LOOPER \\ San Jose State University, San Jose, California 95192
}

\begin{abstract}
This study investigates the influence of attention loading on the established intersensory effects of passive bodily rotation on choice reaction time (RT) to visual motion. Subjects sat at the center of rotation in an enclosed rotating chamber and observed an oscilloscope on which were, in the center, a tracking display and, $10^{\circ}$ left of center, a RT line. Three tracking tasks and a no-tracking control condition were presented to all subjects in combination with the RT task, which occurred with and without concurrent cab rotations. Choice RT to line motions was inhibited $(p<.001)$ both when there was simultaneous vestibular stimulation and when there was a tracking task; response latencies lengthened progressively with increased similarity between the RT and tracking tasks. However, the attention conditions did not affect the intersensory effect; the significance of this for the nature of the sensory interaction is discussed.
\end{abstract}

Human processing of visual-vestibular information is an important area for investigation, since these two systems are known to be interrelated in significant ways, with their converging sensory inputs providing stability to the perceived, spatial world. Congruent vestibular information facilitates performance on particular visual sensorimotor tasks, whereas disruption in the correspondence between the information conveyed by these senses can produce performance degradation (Beck, 1974; Guercio \& Wall, 1972), subjective disorientation, and has even been suggested as the cause of motion sickness (Reason, 1969).

Under appropriate conditions, activity associated with either the vestibular or ocular system induces activity in the other. For example, under certain circumstances, ocular nystagmus and the oculogyral illusion are activated by stimulating the semicircular canals (Guedry, 1965). Similarly, sensitivity to rotation is enhanced in the presence of static visual stimuli as compared to thresholds measured in complete darkness (Clark \& Stewart, 1972). Furthermore, nonveridical perception of self-rotation, "circularvection," can be produced by appropriate visual motion stimulation (Young, Dichgans, Murphy, \& Brandt, 1973). Electrophysiological studies have demonstrated that, during circularvection, evoked activity occurs in neurons in the vestibular nuclei of

This study was conducted under NASA Grant NGL 05-046002 to San Jose State University at NASA Ames Research Center, Moffett Field, California. Brant Clark and John Stewart were closely involved with the project in every phase, from its conception through its completion. Their knowledge, experience, and technical expertise have been invaluable. Requests for reprints may be sent to Brant Clark at the Department of Psychology, San Jose State University, San Jose, California 95192. the brainstem and in the eighth nerve that resembles "the effect of an adequate stimulation of the semicircular canals" (Young et al., 1973, p. 30). Dichgans and Brandt (1972) have suggested that "visual information about motion of one's surroundings may be evaluated by central nervous structures that normally process vestibular input" (p. 327). And, according to Jung (1961), onset and/or cessation of electrical polarization of the vestibular apparatus activates nearly all the neurons of the visual cortex.

Recently, visual and vestibular sensory-interaction effects analogous to those known between the visual and auditory systems have been observed in psychophysical studies. However, while an auditory stimulus typically facilitates (shortens) reaction time (RT) to a visual cue (e.g., Bernstein, Rose, \& Ashe, 1970), vestibular stimulation usually inhibits (lengthens) choice RT to visual motion (Borkenhagen, 1974; Clark \& Stewart, 1974a, b; Mattson, 1975; Stewart \& Clark, Note 1). All but one of these studies used discrete pulses of paired intermodal stimuli under relatively uncomplicated perceptual circumstances.

The purpose of the present study was to investigate the effect of a complex psychomotor situation on the visual-vestibular bisensory effect. Attention loading (a selection of tracking tasks), which basically has the effect of diminishing the effectiveness of perceptually peripheral stimuli (e.g., Webster \& Haslerud, 1964), was employed. Whether simultaneous bodily rotation would affect choice RT to intermittent, lateral accelerations of a vertical line in the subject's visual periphery in a consistent manner while he was also continuously engaged in different compensatory tracking tasks was the primary question.

The single-axis tracking tasks were varied along the 
dimensions of vertical or horizontal direction and moving base (with congruent cab motions) or fixed base (without cab motions). The RT line always moved laterally. In the vertical tracking condition, then, its line of motion was discrepant with that of the tracking cursor; in the two horizontal tracking conditions, both lines moved laterally. Peripheral motion information was expected to be processed differently depending on the presence or absence of this discrepancy of orientations. Increasing similarity between the two tasks should impede detection of the accelerations of the RT line. Should this be the case, the question was then whether the intersensory effect, in turn, would be differentially affected. The effects of two different levels of linerotary acceleration on the sensory interaction were also assessed.

\section{METHOD}

\section{Subjects}

Ten commercial airline pilots were paid to serve as subjects for the study. Their sensitivities to rotation were predetermined and fell within the normal range; all had participated in previous rotation studies.

\section{Apparatus}

Body-rotation stimuli. The specially designed, one-degree-offreedom Ames Man-Carrying Rotation Device (MCRD) delivered the vibration-free, semicircular-canal stimulations (Clark \& Stewart, 1968). This device consists of an enclosed chamber, insultated from such external cues as light and sound. The subject sat at the center of rotation, secured by a seat-belt and shoulder harnesses, his aviator's helmet held firmly in a headrest on the back of the seat. Thus linear acceleration was negligible. A voice communication system linked him and the control room.

Visual stimuli. Two movable visual stimuli (cursors) appeared on a cathode ray tube positioned in the MCRD approximately $66 \mathrm{~cm}$ in front of the subject at eye level. These were to represent objects exterior to the vehicle. A third, fixed visual stimulus, the reference cross, was to represent the vehicle's nose. The luminosities of the visual stimuli were equal and provided high contrast with the background screen (in excess of 1,000 times). Thus, the display consisted of two independent parts: a choice RT display and a tracking cursor and reference cross. (a) $R T$ stimuli. In all conditions, a vertical line $10^{\circ}(175 \mathrm{mrad})$ left of the center of the oscilloscope was used to assess choice RT. This line was $1.5 \mathrm{~mm} \times 8.5 \mathrm{~cm}$, could be accelerated laterally in either direction, and continued to accelerate until subject responded, at which time it instantly returned to the starting position. There were two RT stimulus conditions: line motion alone and line motion plus simultaneous cab rotations. Visual motion onset, offset, and rise times were adjusted to match those for the rotary stimuli. Visual and rotary movements were always in opposite directions with acceleration levels equal, simulating the real-life situation. (b) Tracking stimuli. At the center of the screen, also in all conditions, was a fixed reference cross with each arm, $1.5 \mathrm{~mm} \times 1.5 \mathrm{~cm}$, that indicated the center of the tracking display. During any condition which involved a tracking task there was also a cursor, i.e., the moving line which the subject tracked. The cursor was $1.5 \mathrm{~mm} \times 8.5 \mathrm{~cm}$ and was positioned either vertically or horizontally, depending on the tracking task. When the cursor was vertical, it moved laterally (horizontal tracking); when it was horizontal, it moved vertically (vertical tracking). Moving-base tracking involved cab rotations corresponding to the motions of the tracking cursor.
An analog computer controlled the motions of the MCRD and of the visual displays. The motions of the tracking cursor and the related cab movements were determined by the tracking error, which was computed as the difference between the forcing function, tape-recorded sum-of-10 sine waves with frequencies from 0.1 to $11.6 \mathrm{rad} / \mathrm{sec}$, and the computed simulated aircraft response to the pilots' control inputs. The simulated aircraft dynamics required the pilot to operate as an acceleration controller, i.e., the displacement of the tracking control commanded aircraft attitude acceleration. It should be noted that when the tracking task occurred with congruent MCRD motion and the RT task also involved cab motion, the latter motion was superimposed on the former until the subject responded. This means that the cab rotations associated with the RT task were randomly modified very slightly in this condition. However, due to the high frequencies of the sine waves associated with the tracking task, they functioned as mere noise and were not sufficient to mask the constant acceleration of the cab in the opposite direction to that of the RT line.

Response apparatus. The subject could change the position of the tracking cursor by moving a fingertip controller on the front of the right armrest forward and back for vertical tracking or from side to side for horizontal tracking. The control inputs had to be in the same direction as the motion of the cursor, as would be required in controlling an aircraft with the usual display information. In addition, a control stick located on the front of the left armrest, $8.0 \mathrm{~cm}$ in length, was to be pressed quickly in the same direction as RT line displacement to register choice RT. The switch was activated by a force of $1.1 \mathrm{~N}$.

A time history was kept on an eight-channel ink recorder which charted the directions of RT line displacement and subjects' choice RT responses, tracking errors, squared and integrated every $10 \mathrm{sec}$ and at the end of each run, the forcing function, and the accelerations of the MCRD. Choice RT was displayed in milliseconds on a digital recorder.

\section{Procedure}

There were four basic experimental conditions, each defined by the type of tracking task involved: Condition 1, no tracking: Condition 2, vertical, fixed-base tracking: Condition 3, horizontal, fixed-base tracking: and Condition 4, horizontal, movingbase tracking (previously defined under tracking stimuli in the Apparatus section). Within each tracking condition, there were blocks of two RT conditions (line accelerations with and without opposite rotary motion) and blocks of two levels of RT linerotary acceleration, $1 \% / \mathrm{sec}^{2}$ and $6^{\circ} / \mathrm{sec}^{2}\left(17.5 \mathrm{mrad} / \mathrm{sec}^{2}\right.$ and $105 \mathrm{mrad} / \mathrm{sec}^{2}$, respectively). Each subject completed a full experimental condition, consisting of one tracking task, both RT conditions, and both levels of acceleration, before proceeding to another tracking condition. Different presentation orders of the tracking blocks, RT conditions, and acceleration levels were assigned randomly to the subjects to mitigate order effects.

The basic task was to perform continuous, compensatory tracking, attempting to maintain congruency between the moving cursor and the fixed cross as nearly as possible, and, further, to respond immediately and accurately in the direction of the acceleration of the vertical line in the visual periphery whenever this occurred. There were no instructions regulating the subject's eye movements during the tracking; presumably, the efficient execution of the tracking task would require that the subject attend to the cursor and the reference cross continuously. During the no-tracking condition, the subject was instructed to direct his eyes toward the central cross at all times during the experimental session.

On the subjects' first day, each was given time to learn the basic tracking and choice RT task. A preliminary study had shown that there was positive transfer of learning from each type of tracking to the others, so each subject was trained on only one of the three tasks during this time. The type of tracking during this initial phase was randomly assigned to the subjects, 
with the restriction that each type be practiced during this time by at least three persons. The training period consisted of three 3-min tracking runs followed by three 3-min runs that combined tracking with the choice RT task. Rests of 1.5 min duration were given between runs. During the combination runs, each RT condition was practiced separately. Only one of the two acceleration levels was used per subject, with half of them assigned to $1^{\circ} / \mathrm{sec}^{2}\left(17.5 \mathrm{mrad} / \mathrm{sec}^{2}\right)$ and half to $6^{\circ} / \mathrm{sec}^{2}\left(105 \mathrm{mrad} / \mathrm{sec}^{2}\right)$. This extended training period occurred once only for each subject at the beginning of his participation in the study.

After this initial training, whenever a pilot was tested, he began with two 3-min practice periods that combined the particular tracking task associated with the experimental condition and the choice RT task. During the no-tracking condition, choice RT was practiced alone. Again, as in the initial training, both RT conditions but only one of the acceleration levels were practiced, with the latter randomly assigned to the subjects and half the subjects practicing each.

Following practice, there were four experimental runs consisting of one tracking condition with each of two RT conditions combined with each of the two levels of acceleration. Each run lasted approximately $190 \mathrm{sec}$ or long enough to collect 10 choice RTs. Throughout, 1.5-min rests were provided between runs. All subjects performed each of the experimental task situations.

At the beginning of each session, the subject fixed his eyes on the reference cross, answered a ready signal when he was prepared, and began the tracking task. After 2 or $3 \mathrm{sec}$, there was an acceleration of the RT line (with or without rotary motion, depending on the condition), which was interrupted and returned to position by the subject's response to it. Thereafter, at 20 -sec intervals, line motions occurred until the series was completed.

\section{RESULTS}

\section{Tracking Performance}

As anticipated, there was a marked positive transfer of learning from each tracking task to the others, supporting the contention that preliminary training on only one would be sufficient for asymptotic performance during experimental trials. There were no obvious differences in the difficulty of learning among the types of tracking.

Figure 1 displays the learning curve consisting of the mean square tracking error pooled across subjects and across tracking conditions for training, practice, and experimental trials. Errors decreased sharply, initially,

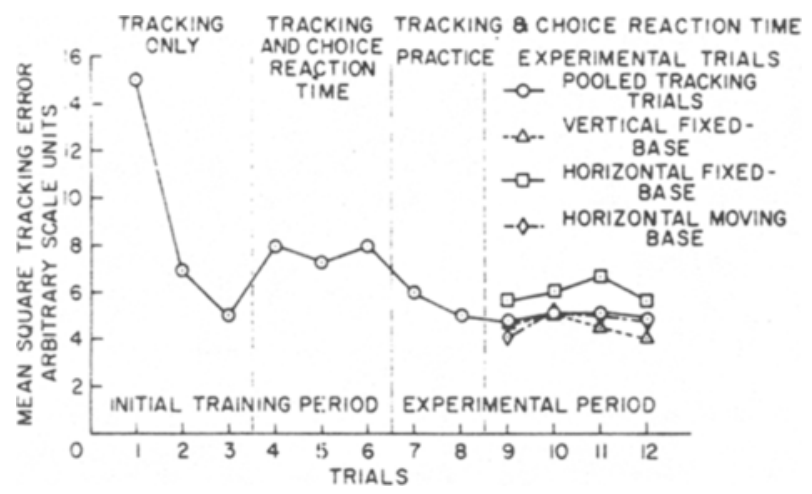

Figure 1. Mean square tracking error pooled across 10 subjects for training, practice, and experimental trials.

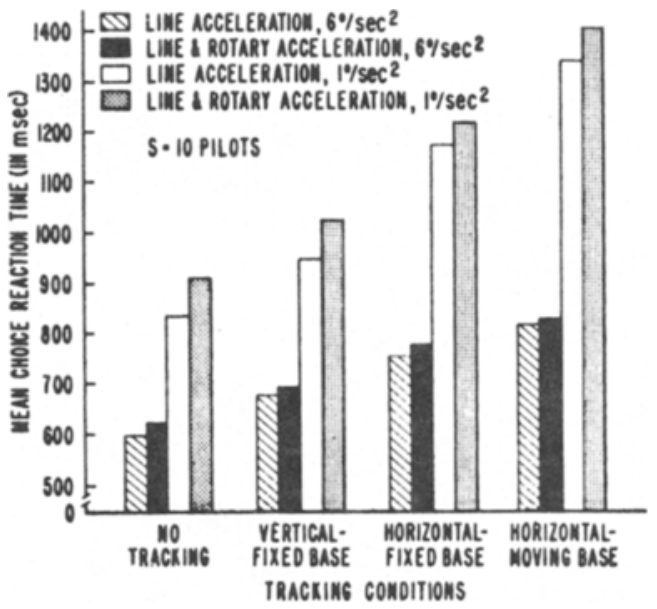

Figure 2. Mean choice reaction time for four tracking conditions, two levels of acceleration, and two motion conditions.

and at the end of the third trial, after approximately 10 min of practice, there had been marked error reduction. When, at the fourth trial, the second task, choice $\mathrm{RT}$, was introduced in addition to the tracking, errors again increased, as would be expected. It was during the two practice trials of the experimental period that the effect of positive leaming transfer was clearly evidenced by the immediate diminution of tracking error even though the subject now performed a new tracking task (Trials 7 and 8, Figure 1). By the ninth trial, the first of the experimental series, tracking error had become relatively stable and remained consistent throughout the remainder of the trials. Also included in Figure 1 are curves representing mean square tracking error across subjects for each tracking condition during the four experimental trials, which illustrate that performance on each loading task was asymptotic during these trials.

\section{Reaction Time Performance}

Errors in choice RT were low in frequency, $1.7 \%$. These trials were not repeated, since this would necessarily have resulted in further practice of both choice RT and tracking for subjects making errors, which was deemed a greater possible problem to the study than errors in RT. Since an inspection of the data revealed no obvious differences between choice RTs involving errors and those not in error, and since these were few in number, these times were included in the data analysis. Thus, the mean RTs of individual subjects reflected the same number of trials each.

Ten choice RTs per condition were recorded for each subject, with the first response treated as practice and not included in the data analysis. The means of the remaining nine were subjected to a four-way factorial analysis of variance with repeated measures. Figure 2 graphically presents group mean choice RT for all experimental conditions; means and standard deviations are in Table 1 . There was a wide range in mean choice RT, from $602 \mathrm{msec}$ in the no-tracking, no-rotation, higher-intensity condition to $1,406 \mathrm{msec}$ in the 
Table 1

Group Mean Choice Reaction Time in Milliseconds and Standard Deviations

\begin{tabular}{|c|c|c|c|c|c|}
\hline \multirow{2}{*}{$\begin{array}{l}\text { Tracking } \\
\text { Conditions }\end{array}$} & & \multicolumn{2}{|c|}{$\begin{array}{c}\text { Level of Acceleration } \\
1 \mathrm{Deg} / \mathrm{Sec}^{2}\end{array}$} & \multicolumn{2}{|c|}{$\begin{array}{c}\text { Level of Acceleration } \\
6 \mathrm{Deg} / \mathrm{Sec}^{2}\end{array}$} \\
\hline & & No Rotation & Rotation & No Rotation & Rotation \\
\hline No Tracking & $\begin{array}{l}\text { Mean } \\
\text { SD }\end{array}$ & $\begin{array}{l}838 \\
110\end{array}$ & $\begin{array}{r}912 \\
86\end{array}$ & $\begin{array}{r}602 \\
60\end{array}$ & $\begin{array}{r}626 \\
73\end{array}$ \\
\hline Vertical Fixed Base & $\begin{array}{l}\text { Mean } \\
\text { SD }\end{array}$ & $\begin{array}{l}947 \\
138\end{array}$ & $\begin{array}{r}1025 \\
151\end{array}$ & $\begin{array}{r}682 \\
58\end{array}$ & $\begin{array}{r}692 \\
88\end{array}$ \\
\hline Horizontal Fixed Base & $\begin{array}{l}\text { Mean } \\
\text { SD }\end{array}$ & $\begin{array}{r}1174 \\
193\end{array}$ & $\begin{array}{c}1218 \\
227\end{array}$ & $\begin{array}{l}755 \\
104\end{array}$ & $\begin{array}{l}781 \\
103\end{array}$ \\
\hline Horizontal Moving Base & $\begin{array}{l}\text { Mean } \\
\text { SD }\end{array}$ & $\begin{array}{r}1341 \\
192\end{array}$ & $\begin{array}{r}1406 \\
244\end{array}$ & $\begin{array}{l}819 \\
112\end{array}$ & $\begin{array}{l}828 \\
106\end{array}$ \\
\hline
\end{tabular}

horizontal, moving-base, rotation, lower-intensity condition.

The analysis of variance revealed that all the main effects were highly significant: tracking conditions, $F(3,27)=51.15, \quad p<.001, \quad$ RT conditions, $F(1,9)=29.56, p<.001$, and levels of acceleration, $F(1,9)=302.97, p<.001$. The shortest choice RTs were elicited during the no-tracking condition, the longest, during horizontal, moving-base tracking. Medium choice RTs occurred during the fixed-base conditions, with RTs associated with vertical tracking shorter than those associated with horizontal tracking. Group mean choice RT to the bimodal stimulus was consistently longer than those to the visual stimulus alone, and choice RTs were slower at $1 \% / \mathrm{sec}^{2}$ $\left(17.5 \mathrm{mrad} / \mathrm{sec}^{2}\right)$ than at $6 \% / \mathrm{sec}^{2}\left(105 \mathrm{mrad} / \mathrm{sec}^{2}\right)$.

There were also two significant interactions, the first being between tracking conditions and levels of acceleration, $F(3,27)=18.06, p<.001$, and the second, between RT conditions and levels of acceleration, $F(1,9)=$ $5.29, p<.05$. The low-acceleration RT stimulus was more susceptible to influence by both tracking conditions and body rotation. Importantly, there was no interaction between RT conditions and tracking conditions, $F(3,27)=0.16$, n.s.

\section{DISCUSSION}

There are two basic results in this study. First, the progressive lengthening of RTs over tracking conditions demonstrates that, as expected, the degree of similarity between two sets of stimuli (in this case, those associated with the tracking task and those associated with the choice RT task) greatly affects the ease or difficulty of perceptually separating the two. When the tracking task was vertical, and the cursor and the RT line movements were oriented differently within the frontoparallel plane, the horizontal accelerations of the RT line were more easily detected. When, however, their motions were in the same orientation, i.e., both horizontal, RT to the peripheral line was impeded. The greatest hindrance to RT occurred when there were accelerations of the cab accompanying both the visual tracking and the RT line, at which time the number of sameorientation directional cues were maximal. The highly significant interaction between tracking conditions and levels of acceleration (see Figure 2) indicates that the peripheral line accelerations at the lower intensity were especially difficult to discern during horizontal tracking.

Second, of primary interest in the study is the present result of inhibition of choice $R T$ to visual motion by simultaneous vestibular stimulation, which corroborates the findings of other visual-vestibular experiments (Borkenhagen, 1974; Clark \& Stewart, 1974a, b; Mattson, 1975; Stewart \& Clark, Note 1). Although one study (Lee, 1973) found no inhibition in a particular experimental situation, the preponderance of evidence supports the findings of the present inquiry. The effect occurred not only in the no-tracking condition but when there was attention loading as well. In spite of the fact that the different combinations of tasks proved to be of varying difficulty, the degree of the intersensory inhibition did not differ over these various conditions; i.e., it was not affected by differential attention loading. The implications of this result will be discussed below.

A stimulus may be processed by the nervous system as energy, as information, or both (Gamer, 1974; Gibson, 1966). The suggestion here is that an extramodal, auxiliary stimulus may exert its influence through either its energic aspect (i.e., its intensity), its information content, or a combination of these factors. For example, if the information of the auxiliary stimulus (in this study, body rotation via vehicle rotation) were unexpected or contradictory to that of the primary stimulus (e.g., the acceleration of the RT line), interference with the task should accrue. However, when its information is congruent, supplementary, or redundant to that of the primary stimulus, facilitation, if anything, should occur (unless bimodal stimuli are processed successively in this situation).

The information of the vestibular stimuli was redundant to that of the visual stimuli in the present study. Although visual and vestibular cues were always in opposite directions, this simulates the real-life situation in which the visual stimuli on the oscilloscope (which represented static exterior cues) would appear to move 
in opposition to the vehicle motions. Therefore, in terms of the information content, the rotary cues should have facilitated performance of both tasks. In fact, although not statistically tested, tracking-related vehicle movements probably did facilitate subjects' tracking (cf. horizontal, fixed- and moving-base tracking, Figure 1); however, the bodily rotation simultaneous to the acceleration of the RT line interfered with visual choice RT, as predicted. The inhibitory effect was less at the higher level of acceleration of the line and the cab; perhaps the redundancy of the vestibular information was at this time more readily perceived and utilized.

Then, the intersensory effect may be due to a direct inhibitory influence by the energy of the vestibular activity upon structures processing the visual motion information. There is evidence that interactions between the afferent impulses of these sensory systems do occur (Gorgiladze \& Smirnov, 1970; Jung, 1961; Medvedeva, 1970). If so, stimulus energy levels would be important; interference with RT to a visual motion stimulus of a constant intensity should be a function of increasing proportions of intensity of the auxiliary vestibular stimulus. The results of two studies did follow this pattern (Clark \& Stewart, 1974a; Mattson, 1975).

Alternatively, if the intersensory inhibition were due to an incongruent, irrelevant, or distracting nature of the auxiliary body-rotation stimulus, the effect (i.e., the differences between visual $\mathrm{RT}$ in the rotation and norotation conditions) should have been augmented as the difficulty of the combined tasks increased. However, this did not occur (Figure 2); in fact, the interaction between RT conditions and tracking conditions was near zero.

Because the line and rotary motions were in opposite directions, there is a third possible explanation of the interference effect, that of response conflict. However, this, too, is unlikely, since Clark and Stewart (1974a, b) have shown that inhibition of visual RT occurs even when the motions are in the same direction. Also, in the present study, there were no false RTs in the absence of line motions in the moving-base tracking condition, as might have occurred if the subjects were confused by cab rotations. Nor did an analysis of variance of number of errors in the direction of RT responses reveal a difference between the rotation and no-rotation conditions, $F(1,9)<1$, n.s.

\section{REFERENCE NOTE}

1. Stewart, J. D., \& Clark, B. Choice reaction time to visual motion during prolonged rotary motion in airline pilots. Paper presented at the Aerospace Medicine Meeting, Washington, D.C., May 1974.

\section{REFERENCES}

BECK, L. J. The effect of spurious angular accelerations in dynamic simulation. Human Factors, 1974, 16, 423.431.
Bernstein, I. H., Rose. R., \& Ashe, V. M. Energy integration in intersensory facilitation. Joumal of Experimental Psychology, 1970, 86, 196-203.

Borkenhagen, J. M. Rotary acceleration of a subject inhibits choice reaction time to motion in peripheral vision. Journal of Experimental Psychology, 1974, 102, 484-487.

Clark, B., \& Stewart, J. D. Comparison of three methods to determine thresholds for perception of angular acceleration. American Joumal of Psychology, 1\%8, 81, 207-216.

Clark, B., \& Stewart, J. D. Comparison of the sensitivity to rotation of pilots and nonpilots. Aerospace Medicine, $1972,43,8-12$.

Clark, B., \& Srewart, J. D. Choice reaction time to visual motion with varied levels of simultaneous rotary motion. American Journal of Psychology, 1974, 87, 441-448. (a)

Clark, B., \& Stewart, J. D. Effects of angular acceleration on man: Choice reaction time using visual and rotary information. Perceptual and Motor Skills, 1974, 38, 735-743. (b)

Dichgans, J., \& Brandt, T. Visual-vestibular interaction and motion perception. In J. Dichgans \& E. Bizzi (Eds.), Cerebral control of eye movements and motion perception. Basel: S. Karger, 1972.

GARNER. W. R. The processing of information and structure. New York: Halsted Press, 1974.

GiBson, J. J. The senses considered as perceptual systems. Boston: Houghton Mifflin, 1966.

Gorgiladze, G. I., \& Smirnov, G. D. Electrophysiological investigation of the interaction of the vestibular and visual afferent systems. In V. V. Parin \& M. D. Yenielyanov (Eds.), Physiology of the vestibular analyzer. Washington, D. C: NASA Technical Translation TT-F616, June 1970.

Guedry, F. E. Studies of vestibular function. In W. D. Neff (Ed.), Contributions to sensory physiology. New York: Academic Press. 1965.

Guercio, J. G.. \& Wall, R. L. Congruent and spurious motion in the learning and performance of a compensatory tracking task. Human Factors, 1972, 14, 259-269.

Jung, $R$. Neuronal integration in the visual cortex and its significance for visual information. In W. A. Rosenblith (Ed.). Sensory communication. New York: Wiley, 1961.

LEE. A. T. Effects of tracking task load and angular acceleration on choice reaction time to peripheral motion. Unpublished masters thesis, California State University. San Jose, August 1973.

Matsson, D. L. Visual reaction times during prolonged angular acceleration parallel the subjective perception of rotation. Journal of Experimental Psychology: Human Perception and Performance, 1975, 104, 404-410.

Medvedeva, N. G. On the problem of the interaction mechanism of the visual and vestibular analyzers. In V. V. Parin \& M. D. Yeniel'yanov (Eds.), Physiology of the vestibular analyzer. Washington, D.C: NASA Technical Translation TT-F616, June 1970.

ReAson, J. L. Mution sickness-some theoretical considerations. International Journal of Man-Machine Studies, $1969,1,21-38$.

Webster, R. G., \& Haslerud, G. M. Influence on extreme peripheral vision of attention to a visual or auditory task. Journal of Experimental Psychology, 1964, 68, 269-272.

Younc, L. R., Dichgans, J., Murphy, R., \& Brandt, L. Interaction of optokinetic and vestibular stimuli in motion perception. Acta Otolaryngologica, 1973, 76, 24-31. 